\title{
A detecção da aterosclerose subclínica pode modificar o risco de desenvolvimento da doença cerebrovascular?
}

\author{
The subclinical atherosclerosis can change the risk of cerebrovascular \\ disease?
}

Elizabete V. de Freitas ${ }^{\star}$

\section{Resumo}

A literatura científica tem reconhecido que a doença cerebrovascular pode causar déficit cognitivo. A demência vascular é melhor compreendida como uma síndrome heterogênea do que como uma doença distinta. Em todo o mundo aproximadamente 35,6 milhões de pessoas vivem com demência e a projeção é que esse número duplique em 2030. A Organização Mundial de Saúde reconhece a demência como uma prioridade pública. $\mathrm{O}$ acidente vascular cerebral permanece como a primeira causa de incapacidade e a terceira de morte nos Estados Unidos. Um em cada três americanos terá um acidente vascular cerebral ou desenvolverá demência ao longo da vida. A prevalência de doença de Alzheimer dobra a cada 4,3 anos e a demência vascular dobra a cada 5,3 anos. A demência é uma das maiores causas de incapacidade e dependência entre os idosos em o todo mundo. Representa uma grande carga para os doentes, bem como para seus cuidadores e família. Apesar das controvérsias, as evidências de estudos observacionais e clínicos mostraram que os fatores de risco convencionais (hipertensão arterial, diabetes mellitus, dislipidemia, fumo, fibrilação atrial e estilo de vida, por exemplo) desempenham importante papel no desenvolvimento de dano cognitivo e demência. Infelizmente, embora saibamos muito sobre os fatores de risco para a demência, não sabemos como eles interagem, como contribuem ou em que proporção e quais intervenções específicas podem reduzir este risco. Futuras pesquisas são necessárias para esclarecer se a atuação sobre os fatores de risco poderá limitar o dano vascular cerebral e prevenir o déficit cognitivo e a demência.

Descritores: Aterosclerose; Fatores de risco; Doença cerebrovascular; Acidente vascular Cerebral; Demência.

\footnotetext{
Abstract

The scientific literature has recognized that cerebrovascular disease can cause severe cogni-

*Endereço para correspondência: Serviço de Cardiologia, HUPE, UERJ. Boulevard 28 de Setembro, $77,2^{\circ}$ andar Rio de Janeiro, RJ, Brasil. CEP: 20551-030. E-mail: elizabet@rio.com.br
} 
tive deficits. The entity of vascular dementia is best understood as a heterogeneous syndrome than a distinct disorder. Worldwide, nearly 35.6 million people live with dementia. This number is expected to double in 2030. World Health Organization recognizes dementia as a public health priority. Stroke remains the number one cause of disability and the third cause of death in USA. One in three Americans will have a stroke or develop dementia in their lifetime. The prevalence of Alzheimer disease doubles every 4.3 years and that of vascular dementia doubles every 5.3 years. Dementia is one of the major causes of disability and dependency among older people worldwide. It is overwhelming not only for the people who have it, but also for their caregivers and family. There is substantial evidence, despite controversial issues, from observational studies and clinical trials that conventional risk factors such hypertension, diabetes, dyslipidemia, smoking, atrial fibrillation and life style, for example, play a role in the development of cognitive impairment and dementia. This is a field in need of research because further decline in cognition might be preventable in the early stages. Unfortunately, although we now know much about individual risk factor for dementia, we do not know how they interact or which risk factors account for what proportion of dementia cases and which specific interventions can lower this risk. Future research is needed to clarify the role of risk factors modification in limiting vascular brain injury to prevent vascular cognitive impairment and dementia.

Keywords: Atherosclerosis; Risk factors; Cerebrovascular disease; Stroke; Dementia.

\section{Introdução}

Em todo o mundo, aproximadamente 35,6 milhões de pessoas vivem com demência, sendo esperado, segundo os dados da Organização Mundial de Saúde (OMS), que esse número dobre em 2030 (65,7 milhões) e em 2050 atinja mais do triplo (115,4 milhões). ${ }^{1}$
Os dados demográficos e populacionais em todo o mundo indicam que o envelhecimento populacional tem como consequência o aumento do número de pessoas afetadas pela demência. Projeta-se que em 20301 em cada 5 americanos tenha idade igual ou superior a 65 anos e até 2040 esse número atingirá a marca de 1,3 bilhões. Nesse cenário, o transtorno cognitivo e os quadros demenciais representam uma das condições mais temidas. ${ }^{1,2,3}$

A demência afeta pessoas de todos os países, sendo que cerca de $2 / 3$ desses indivíduos vivem em países em desenvolvimento, onde também ocorrerá um aumento mais pronunciado de casos. ${ }^{3,4}$ As estimativas do estudo de consenso Delphi ${ }^{4}$ são assustadoras com um novo caso a cada 7 segundos, ou seja 4,6 milhões a cada ano. Nos EUA, de acordo com Centro de Controle e Prevenção de Doenças, só a doença de Alzheimer foi considerada, em 2007, a sexta causa de óbito em todas as idades. ${ }^{5}$

No Brasil as estatísticas não são exatas, mas estima-se que tenham ocorrido aproximadamente 1,25 milhões de casos de demência em 2010.

O tratamento e os cuidados dispensados a pessoas com demência consomem atualmente mais de 604 bilhões de dólares por ano em todo o mundo, incluindo custos sociais e com a saúde, bem como a perda de renda das pessoas afetadas pela doença e de seus cuidadores. ${ }^{1}$ Essa importância corresponde a $1 \%$ do produto interno bruto (PIB) mundial.

Apesar disso, somente 8 países no mundo têm atualmente um programa nacional direcionado à demência. Os novos relatórios da OMS referem a demência como uma prioridade de saúde pública e recomendam que os programas foquem, de forma incisiva, no diagnóstico precoce e na conscientização pública sobre a doença, reduzindo seu estigma e proporcionando mais e melhor assistência aos cuidadores. ${ }^{1}$

A maioria dos casos não são detectados precocemente, sendo imputados ao processo de envelhecimento sintomas de déficits cognitivos, com negligência na avaliação da função cogni- 
tiva através de exames que devem fazer parte do arsenal de rotina de todos os médicos que tratam de pacientes idosos, especialistas ou não.

Infelizmente, além das limitações diagnósticas, há preconceitos relacionados à expectativa negativa dos pacientes, que frequentemente, ao perceberem déficits cognitivos, tentam minimizá-los para que não sejam notados, e de familiares que acreditam estar frente a uma doença sem qualquer tratamento. ${ }^{1,3}$

\section{Demência}

A demência é uma síndrome crônica de natureza progressiva, na qual há deterioração na função cognitiva que afeta a memória, o pensamento, a orientação, a compreensão, o cálculo, a capacidade de aprendizado, a linguagem e o julgamento, sem comprometimento da consciência. $\mathrm{O}$ déficit cognitivo é habitualmente acompanhado ou precedido por distúrbios no controle emocional, no comportamento social ou motivação. Portanto, caracterizada por afasia (alteração da fala), agnosia (incapacidade de reconhecimento), apraxia (dificuldade na realização de atividade motora) e por comprometimento da função executiva (incapacidade de planejamento, execução e abstração), com nítida perda em relação ao nível anterior. Para o diagnóstico é essencial que os déficits causem significativo prejuízo na realização das atividades profissionais, ocupacionais e sociais do indivíduo. ${ }^{1,3,6}$

Até o momento os fatores de risco não modificáveis para demência são a idade, o sexo feminino a partir dos 80 anos, síndrome de Down e a história familiar positiva para o genótipo da apoliproteína E4 (APO E4). 2,3

Entre os fatores protetores encontram-se o sexo masculino, nível educacional elevado, vida ativa com estimulação cognitiva constante, engajamento em atividades sociais e de lazer, suporte e redes sociais disponíveis, atividade física regular, dieta rica em antioxidantes e vitaminas (E, C, B6, B12 e folato), ausência de traumas cranianos, presença do alelo E2, níveis baixos de colesterol, consumo moderado de vinho tinto e uso de certos medicamentos como estatinas, anti-hipertensivos, terapia de reposição estrogênica, anti-inflamatórios não esteroides, antioxidantes e agonistas de receptores de histamínicos $\mathrm{H} 2{ }^{3}$

A pesquisa sobre as diferentes taxas de demência nas diversas populações é uma abordagem que representa a identificação dos fatores de risco modificáveis e a possibilidade de prevenir esta doença. A principal causa de demência nas nações ocidentais é a doença de Alzheimer, seguida pela doença cerebrovascular. No Japão e possivelmente em outras nações asiáticas, de acordo com o Honolulu-Asia Aging Study, a principal causa em homens é a doença cerebrovascular. ${ }^{7}$ Os diferentes métodos utilizados, bem como as características genéticas, constitucionais, sociais e/ou as diferenças ambientais entre pessoas de diferentes culturas podem contribuir para essa dessemelhança nas taxas. ${ }^{8-11}$

\section{Por que a demência ocorre em} algumas pessoas e não em outras?

Como a hemorragia e a atrofia cerebral, que podem ser observadas em exames de imagem, têm sido ligadas aos fatores de risco vascular in vivo e com patologia vascular observada em necropsia. A injúria cerebrovascular subclínica aumenta o risco de AVC e é um preditor de dano cognitivo vascular. ${ }^{10-12}$

\section{Como minimizar as chances de no} futuro estar frente a frente com a demência?

Nos últimos anos muitos estudos longitudinais têm definido melhor os fatores de risco para os diversos tipos de demência. ${ }^{8,9}$ Além dos fatores de risco não modificáveis, citados anteriormente, os fatores de risco vasculares têm sido ligados à incidência de todas as demências, incluindo a doença de Alzheimer e a demência vascular isoladamente. ${ }^{8-10}$ Naturalmente, assim como a doença vascular e as alterações neurocognitivas, o transtorno cognitivo vascular é mais comum nos idosos. As formações ateromatosas nos vasos intra e extracranial deixam 
o paciente em risco tanto de infarto cerebral subclínico (ICS), como de acidente vascular cerebral (AVC) e doenças de pequenos vasos. A angiopatia amiloide também pode ocorrer, causando danos na substância branca e podendo, por último, causar hemorragia.

Num estudo holandês ${ }^{12}$ a presença de infarto cerebral silencioso em idoso saudável, visto na ressonância magnética, mostrou aumento no risco de desenvolvimento de demência e de declínio abrupto nas funções cognitivas quando comparado com os que não apresentavam tais lesões.

\section{Fatores de risco para declínio cognitivo e demência}

A hipertensão tem sido associada com injúria cerebral subclínica e pode, desta forma, aumentar o impacto na incidência de acidente vascular cerebral e sobre a função cognitiva. Entretanto, há controvérsias quanto à hipertensão arterial ser um fator de risco para demência e declínio cognitivo, com alguns estudos encontrando uma associação positiva e outros não. ${ }^{13}$ Uma recente revisão baseada nos estudos publicados sob o patrocínio da National Institute of Health (NIH) concluiu que as evidências de tal associação é fraca. ${ }^{14}$ Outro estudo com 11.151 participantes baseado numa coorte da população do Atherosclerosis Risk in Communities $(\mathrm{ARIC})^{15}$ avaliou a associação entre fatores de risco cardiovascular em idade entre 46 e 70 anos e o risco de hospitalização devido à demência, e determinou se essa associação era modificada pela idade e a etnia. Neste estudo prospectivo o fumo, a hipertensão e o diabetes mellitus (DM) estavam fortemente associados com o risco subsequente de hospitalização por demência nesta faixa etária. Esses resultados enfatizaram a importância da atuação precoce sobre os fatores de risco para prevenção de demência. ${ }^{15}$

Outro estudo ${ }^{16}$ em indivíduos independentes com idade entre 65 e 84 anos analisou a influência dos fatores de risco vascular (FRVs) e alteração de substância branca relacionadas com a idade sobre a performance neuropsico- lógica, com o intuito de avaliar dano cognitivo adicional pelos FRVs em adição aos efeitos das alterações da substância branca. Essa coorte foi avaliada através de uma bateria de testes neuropsicológicos. Os autores concluíram que as alterações da substância branca se relacionaram com pior performance na função executiva, na atenção e na velocidade. Enquanto que a hipertensão arterial, AVC prévio e DM influenciaram a performance neuropsicológica, independentemente da severidade da alteração da substância branca, reforçando a necessidade do controle dos fatores de risco vascular para prevenir o declínio cognitivo do idoso. ${ }^{16}$

Outros estudos ${ }^{17,18,19,20}$ avaliaram a influência da hipertensão arterial com o declínio cognitivo. Uma coorte do Longitudinal Population Study ${ }^{17}$ mostrou que a hipertensão arterial prévia pode aumentar o risco para demência por induzir doença de pequenos vasos e lesões de substância branca. Também foi observado declínio na pressão arterial anos antes do início da demência, sem que fosse concluído se essa queda é causa ou consequência da doença cerebral. $\mathrm{O}$ Honolulu-Asia Aging Study, ${ }^{18}$ baseado numa comunidade de homens japoneses-americanos livres de demência, avaliou se a pressão de pulso (PP) estava relacionada com o aparecimento de demência. $O$ estudo incluiu 2.505 participantes, com uma média de idade de 57,9 anos (considerada no estudo como meia idade) na primeira avaliação e 76,9 anos na primeira avaliação para demência. A partir de então foram acompanhados por uma média de 5,1 anos. Não houve relação entre demência e nenhum tertil de PP, entretanto a pressão sistólica isolada (PSI) foi o melhor preditor. O estudo concluiu que para homens na meia idade a PSI é o mais forte componente da pressão arterial que prediz a incidência de demência. ${ }^{18}$

A maioria dos estudos sobre a relação entre pressão arterial e cognição foram realizados com coortes de populações de meia idade e idosos; porém em um estudo longitudinal de 20 anos com 529 participantes, constituindo 2 grupos - um de 18 a 46 anos e outro de 47 a 83 
anos, com altos níveis basais de pressão arterial sistólica e diastólica -, ambos os grupos foram fortemente associados a declínio nas habilidades cognitivas. Os resultados sugerem que os adultos jovens, assim como os idosos, são vulneráveis aos efeitos da hipertensão arterial sobre a função cognitiva. ${ }^{21}$

A fibrilação atrial também foi associada ao alto risco de desenvolvimento de demência. ${ }^{2}$ Estudos adicionais são necessários para a avaliação dos tratamentos específicos, como a anticoagulação, que se em nível ideal poderia reduzir este risco.

Apesar das controvérsias, diversos estudos prospectivos têm encontrado associação entre diabetes mellitus (DM) e aumento de risco de declínio cognitivo e demência. ${ }^{22-24}$ Uma revisão sistemática de 14 estudos observou que o DM está associado com o aumento no risco de desenvolvimento de qualquer tipo de demência, incluindo a doença de Alzheimer. ${ }^{25}$ Os achados de acordo com a metodologia dos estudos sugerem que a alteração na glicose, a doença vascular, a insulina e o metabolismo amiloide são elementos da fisiopatologia relevantes, embora seu mecanismo seja incerto, sendo necessários estudos adicionais. ${ }^{25} \mathrm{O}$ estudo da cidade de Vantaa, ${ }^{23}$ na Finlândia, avaliou 553 indivíduos com idade de 85 anos ou mais. O estudo foi iniciado em 1991. Os sobreviventes foram reexaminados em 1994, 1996, 1999 e em 2001. Foram realizadas necropsias em 291 (48\% da população registrada). Os autores concluíram que pacientes idosos com DM desenvolvem patologia vascular mais extensa que sozinha ou associada à doença de Alzheimer, particularmente nos portadores de APOE4, o que resulta em aumento do risco de demência. ${ }^{23}$

Em relação à síndrome metabólica, os dados disponíveis até o momento são inconclusivos, com estudos encontrando relação positiva e outros não.

Diversos estudos prospectivos mostram relação positiva entre o fumo na meia idade e na velhice e o aumento no risco de demência. ${ }^{15,26,27}$ Uma metanálise de 19 estudos com acompa- nhamento de pelo menos 1 ano, concluiu que idosos fumantes apresentam risco de demência por doença de Alzheimer, por doença vascular e qualquer outro tipo de demência, além de associar o fumo com o declínio no desempenho no Miniexame do Estado Mental (MEEM). ${ }^{15,26}$ No Honolulu Heart Program ${ }^{27}$ o fumo foi associado à doença de Alzheimer e a todas as demências combinadas. Nos resultados de necropsias foi observado maior número de placas neuríticas nos que fumavam maior número de cigarros. Entretanto, para os grandes fumantes houve ausência de associação com demência, provavelmente devido à maior mortalidade desse grupo. Dados derivados do Health Survey for England, do Adult Psychiatric Morbidity Survey e The Health Improvement Network estimam que dos 10 milhões de fumantes do Reino Unido cerca de 3 milhões têm evidência de doença mental, tendo sido objeto de um editorial da revista Lancet em março de 2013, o qual mostrou a grande preocupação e relação entre fumo e saúde mental.

Diversos trabalhos buscaram a correlação entre colesterol e o risco do desenvolvimento de demência. ${ }^{15,28,29}$ Alguns trabalhos encontraram uma forte relação entre colesterol elevado e demência nos indivíduos sem a presença do alelo APOE4, provavelmente pela influência desse genótipo na captação do colesterol. ${ }^{28,29}$ Por outro lado, o papel das estatinas na prevenção da demência não foi estabelecido. O MRC/BHF Heart Protection Study concluiu que o uso da sinvastatina atuaria reduzindo o risco cardiovascular, incluindo o risco do desenvolvimento de $\mathrm{AVC},{ }^{30}$ mas sem referencia à doença de Alzheimer ou outros tipos de demência.

Entre as evidências mais aceitas, com relação inversa com a doença de Alzheimer, encontra-se o estilo de vida, considerando-se aí a atividade física, a sociabilidade e a atividade cognitiva, apesar de ainda ser obscura sua real ação. ${ }^{31,32,33,34}$

A aterosclerose tem sido implicada como fator de risco para o desenvolvimento de demência, tanto vascular como por doença de Alzheimer, predominantemente na doença 
aterosclerótica de carótidas. Alguns estudos mostram atenuação dessa associação em acompanhamentos mais longos devido à mortalidade da doença aterosclerótica. ${ }^{35}$

\section{Conclusões}

Embora a doença cardiovascular e cerebrovascular com manifestação clínica sejam fatores de risco estabelecidos para declínio cognitivo e demência, é menos sabido sobre os indivíduos que não apresentam as manifestações clínicas dessas doenças. Alguns estudos, entretanto, encontraram acelerado declínio nos testes de performance de memória, fala e testes de associação semântica e de fluência verbal, em pacientes de casas geriátricas sem manifestações clínicas de doença cardiovascular ou cerebrovascular. ${ }^{36,37} \mathrm{O}$

\section{Tabela 1. Pontos de relevância}

- As alterações vasculares cerebrais não se limitam apenas à demência puramente vascular ou por múltiplos infartos.

- A isquemia e a hemorragia cerebral determinam danos cerebrais e interagem com a DA patologicamente de forma bidirecional.

- As alterações vasculares são evitáveis através da atuação sobre os fatores de risco cardiovascular.

- A idade permanece como o mais forte fator de risco para demência, principalmente por DA.

- O primeiro passo na investigação da DA é conhecer a história familiar.

- Os fatores de risco vascular - hipertensão arterial, diabetes mellitus, fumo, hipercolesterolemia, sedentarismo - têm sido ligados à demência, inclusive à DA e à demência vascular, apesar de faltar consistência em todos os estudos.

- A efetividade do tratamento dessas condições na prevenção das demências ainda não está esclarecida.

- Bateria de testes psicológicos deve ser aplicada na investigação da demência.

- O MEEM deve ser aplicado. Os escores abaixo de 24 pontos são sugestivos de alteração cognitiva, exigindo aprofundamento na investigação.

- Os estudos observacionais sugerem que o estilo de vida, particularmente atividade social, mental e física, estão inversamente associados com o risco de demência.

- Outros fatores como o abuso de álcool, obesidade e depressão podem aumentar o risco para a demência e DA.

DA: doença de Alzheimer;

MEEM: miniexame do estado mental. espessamento médio intimal ou a presença de placas bilaterais das carótidas foi forte preditor de demência.

Os achados da relação da doença aterosclerótica e declínio cognitivo e demência sugerem fortemente que a intervenção precoce sobre as doenças ainda subclínicas e sobre os fatores de risco cardiovascular, poderiam retardar o início dos quadros de demência e de doença de Alzheimer.

\section{Referências}

1. World Health Organization. Dementia cases set to triple by 2050 but still largely ignored. Disponível em: http://www.who.int/mediacentre/news/ releases/2012/dementia_20120411/en/index. html . Acesso em: 1 mar. 2013.

2. Dublin S, Andersson ML, Haneuse SJ, Heckbert S, Crane PK, Breitner CS, et al. Atrial Fibrillation and Risk of Dementia: A prospective Cohort Study. J Am Geriatr Soc. 2011 Aug;59(8):136975. http://dx.doi.org/10.1111/j.15325415.2011.03508.x

3. Machado JC. Doença de Alzheimer. In: Freitas EV; Py L; Cançado FAX; Doll J; Gorzoni M, editores. Tratado de Geriatria e Gerontologia. 3a ed. Rio de Janeiro: Guanabara-Koogan (GEN); 2011. p.178-201.

4. Ferri $C P$, Prince M, Brayne C, Brodaty $H$, Fratiglioni L, Ganguli M, et al. Global Prevalence of dementia: a Delphi consensus study. Lancet. 2005 Dec 17;366(9503):2112-7.

5. B Tejada-Vera J, Xu JQ, Kochanek KD, Murphy SL. National vital statistics reports, v. 58 n.19. Deaths: Final data for 2007. Hyattsville, MD: National Center for HealthStatistics. 2010.

6. Shadlen MF. Risk factors for dementia. UpToDate 2013. Disponível em: http://www.uptodate. com/contents/risk-factors-for-dementia?topic. Acesso em: 8 mar. 2013.

7. White L, Petrovitch H, Ross WG, Masaki KH, Abbott RD, et al. Prevalence of Dementia in Older Japanese-American Men in Hawaii: The Honolulu-Asia Aging Study. JAMA. 1996;276(12):955-60.

8. Patterson C, Feightner J, Garcia A, MacKnight C. General Risk Factor for Dementia a systematic evidence review. Alzheimers Dement. 2007 Oct;3(4):341-7. http://dx.doi.org/10.1016/j. jalz.2007.07.001

9. Patterson C, Feightner JW, Garcia A, Hsiung GY, MacKnight C, Sadovnick AD. Diagnosis and treatment of dementia: 1. Risk assessment 
and primary prevention of Alzheimer disease. CMAJ. 2008 Feb 26;178(5):548-56. http://dx.doi. org/10.1503/cmaj.070796

10. Rincon F, Wright CB. Vascular cognitive impairment. Curr Opin Neurol. 2013 Feb;26(1):29-36. http://dx.doi.org/10.1097/ WCO.0b013e32835c4f04

11. Debette S, Seshadri S, Beiser A, Au R, Himali JJ, Palumbo C, et al. Midlife vascular risk factor exposure accelerates structural brain aging and cognitive decline. Neurology. 2011 Aug 2; 77 (5): 461-8. http://dx.doi.org/10.1212/ WNL.0b013e318227b227

12. Vermeer SE, Prins ND, den Heijer T, Hofman A, Koudstaal PJ, Breteler MM. Silent Brain Infarcts and the Risk of Dementia and Cognitive Decline N Engl J Med. 2003 Mar 27;348(13):1215-22.

13. Goldstein FC; Levey AI, Steenland NK. High Blood Pressure and Cognitive Decline in Mild Cognitive Impairment. J Am Geriatr Soc. 2013 Jan;61(1):67-73. http://dx.doi.org/10.1111/ jgs.12067

14. Williams JW, Plassman BL, Burke J, Benjamin S. Preventing Alzheimer Disease and Cognitive Decline. Evid Rep Technol Assess (Full Rep) 2010 Apr;(193):1-727.

15. Alonso A, Mosley TH Jr, Gottesman RF, Catellier D, Sharrett AR, Coresh J. Risk of dementia hospitalisation associated with cardiovascular risk factors in midlife and older age: the Atherosclerosis Risk in Communities (ARIC) study. J Neurol Neurosurg Psychiatry.2009 Nov;80(11):1194-201. http://dx.doi.org/10.1136/ jnnp.2009.176818

16. Verdelho A, Madureira S, Ferro JM, Basile AM, Chabriat H, Erkinjuntti T, et al. Differential impact of cerebral white matter changes, diabetes, hypertension and stroke on cognitive performance among non-disabled elderly. The LADIS study. J Neurol Neurosurg Psychiatry. 2007 Dec;78(12):1325-30.

17. Skoog I, Lernfelt B, Landahl S, Palmertz B, Andreasson LA, Nilsson L, et al. 15-year longitudinal study of blood pressure and dementia. Lancet. 1996 Apr 27;347(9009):1141-5.

18. Freitag MH, Peila R, Masaki K, Petrovitch $\mathrm{H}$, Ross GW, White LR, et al. Midlife pulse pressure and incidence of dementia: the Honolulu-Asia Aging Study. Neurobiol Aging. 2000 Jan-Feb;21(1):49-55.

19. Obisesan TO, Obisesan OA, Martins S, Alamgir L, Bond V, Maxwell C, et al. High blood pressure, hypertension, and high pulse pressure are associated with poorer cognitive function in persons aged 60 and older: the Third National Health and Nutrition Examination Survey. J Am Geriatr Soc. 2008 Mar;56(3):501-9. http://dx.doi. org/10.1111/j.1532-5415.2007.01592.x

20. Tzourio C, Dufouil C, Ducimetière P, Alpérovitch A. Cognitive decline in individuals with high blood pressure: a longitudinal study in the elderly. EVA Study Group. Epidemiology of Vascular Aging. Neurology. 1999 Dec 10;53(9):1948-52.

21. Elias PK, Elias MF, Robbins MA, Budge MM. Blood pressure-related cognitive decline: does age make a difference? Hypertension. 2004 Nov;44(5):631-6.

22. Ott A, Stolk RP, van Harskamp F, Pols HA, Hofman A, Breteler MM. Diabetes mellitus and the risk of dementia: The Rotterdam Study. Neurology. 1999 Dec 10;53(9):1937-42

23. Ahtiluoto S, Polvikoski T, Peltonen M, Solomon A, Tuomilehto J, et al. Diabetes, Alzheimer disease, and vascular dementia: a populationbased neuropathologic study. Neurology. 2010 Sep 28;75(13):1195-202. http://dx.doi. org/10.1212/WNL.0b013e3181f4d7f8

24. Ohara T, Doi Y, Ninomiya T, Hirakawa Y, Hata J, Iwaki T, et al. Glucose tolerance status and risk of dementia in the community: the Hisayama study. Neurology 2011 Sep 20;77(12):1126-34. http://dx.doi.org/10.1212/ WNL.0b013e31822f0435

25. Biessels GJ, Staekenborg S, Brunner E, Brayne C, Scheltens P. Risk of dementia in diabetes mellitus: a systematic review. Lancet Neurol. 2006;5(1):64-74.

26. Ulrich J, Johannson-Locher G, Seiler WO, Stähelin HB. Does smoking protect from Alzheimer's disease? Alzheimer-type changes in 301 unselected brains from patients with known smoking history. Acta Neuropathol 1997;94(5):450-4.

27. Tyas SL, White LR, Petrovitch H, Webster Ross G, Foley DJ, Heimovitz HK, et al. Mid-life smoking and late-life dementia: the HonoluluAsia Aging Study. Neurobiol Aging. 2003 JulAug;24(4):589-96.

28. Solomon A, Kåreholt I, Ngandu T, Winblad B, Nissinen A, Tuomilehto J Serum cholesterol changes after midlife and late-life cognition: twenty-one-year follow-up study. Neurology. 2007;68(10):751-6.

29.Hall K, Murrell J, Ogunniyi A, Deeg M, Baiyewu O, Gao S, et al. Cholesterol, APOE genotype, and Alzheimer disease: an epidemiologic study of Nigerian Yoruba. Neurology. 2006;66(2):2237.

30. Heart Protection Study Collaborative Group. MRC/BHF Heart Protection Study of cholesterol lowering with simvastatin in 20,536 high-risk individuals: a randomised placebo-controlled trial. Lancet. 2002 Jul 6;360(9326):7-22. 
31. Simonsick EM. Fitness and cognition: encouraging findings and methodological considerations for future work. J Am Geriatr Soc. 2003;51(4):570-1.

32. Coyle JT. Use it or lose it--do e6ortful mental activities protect against dementia? N Engl J Med. 2003 Jun 19;348(25):2489-90.

33. Fratiglioni L, Paillard-Borg S, Winblad B. An active and socially integrated lifestyle in late life might protect against dementia. Lancet Neurol. 2004 Jun;3(6):343-53.

34. Lautenschlager NT, Cox KL, Flicker L, Foster JK, van Bockxmeer FM, Xiao J. Effect of physical activity on cognitive function in older adults at risk for Alzheimer disease: a randomized trial. JAMA. 2008 Sep 3;300(9):1027-37. http://www. doi.org/10.1001/jama.300.9.1027

35. van Oijen M, de Jong FJ, Witteman JC, Hofman A, Koudstaal PJ, Breteler MM. Atherosclerosis and risk for dementia. Ann Neurol. 2007 May;61(5):403-10.

36. Wendell CR, Zonderman AB, Metter EJ, Najjar SS, Waldstein SR. Carotid intimal medial thickness predicts cognitive decline among adults without clinical vascular disease. Stroke. 2009;40(10):3180-5. http://www.doi.org/ 10.1161/STROKEAHA.109.557280

37. Wendell CR, Waldstein SR, Ferrucci L, O'Brien RJ, Strait JB, Zonderman AB. Carotid atherosclerosis and prospective risk of dementia. Stroke. 2012 Dec;43(12):3319-24. 